\title{
Transmissibility-based operational modal analysis: Enhanced stabilisation diagrams
}

\author{
Gert De Sitter*, Christof Devriendt and Patrick Guillaume \\ Vrije Universiteit Brussel, Brussel, Belgium
}

\begin{abstract}
Recently it has been shown that also transmissibilities can be used to identify the modal parameters. This approach has several advantages: because of the deterministic character of the transmissibility functions, the estimated parameters are more accurate than the results obtained with the power spectra based operational modal analysis techniques. Another advantage is that the transmissibility functions do not depend on the colouring of the unknown forces. A disadvantage of the transmissibility based operational modal analysis techniques is that non-physical modes show up in the stabilisation diagrams. In this contribution it will first be shown that those non-physical modes will show up when traditional stabilisation diagrams are used. In a second step, a new approach of selecting the physical modes out of a set of estimated modes will be discussed and the new approach will be validated using data generated with an acoustical Finite Element Model. Finally, the approach will be validated using real acoustical data.
\end{abstract}

Keywords: Operational modal analysis, transmissibilities, stabilisation diagrams

\section{Introduction}

The purpose of Operational Modal Analysis (OMA) is to identify the modal parameters using output only data [1-4]. Since the inputs are unknown, it is obvious that additional assumptions are necessary to be able to estimate the modal parameters. For example, until recently all procedures had to assume that the output only data was the response of a system that was excited by white noise input signals. Most of the existing time-domain techniques, e.g. the autoregressive moving averaging based modal analysis, the natural excitation technique (NExT) and stochastic subspace based modal analysis [1,2], make this assumption. Also the well-known frequency domain OMA techniques, e.g. the auto and cross-power based OMA and the Frequency Domain Decomposition technique take this assumption for granted [3-5].

In this paper, a recently developed frequency domain modal analysis technique will be studied: transmissibility based OMA [6]. In the field of modal analysis, transmissibility functions have almost never been used. The concept of transmissibility functions will be recapitulated in Section 2.

Recently, for OMA, it has been found that combinations of the transmissibility functions can be used to estimate the poles and the mode shapes. The only requirement is that the loading cases should change between the measurement sets. A big advantage of the transmissibility based operational modal analysis technique is that the assumtion that the inputs should be white noise signals, is no longer needed [7]. This is a real advantage, since in a lot of applications the input signals contain harmonic components. Another advantage of transmissibility driven operational modal analysis is that dividing the spectra results in smoother functions (i.e. the transmissibility functions) compared to the power spectra that result from multiplying the noisy spectra. It has been shown already on structural data that this results in better estimates [6]. In this paper the transmissibility approach will be used for the first time

*Corresponding author: Gert De Sitter, Vrije Universiteit Brussel, Pleinlaan 2, 1050 Brussel, Belgium. E-mail: gert.de.sitter@vub.ac.be. 
on acoustical data. And also here it will be concluded that the transmissibility approach results in better estimates of the modal parameters compared to the power spectra approach.

A disadvantage of the current transmissibility based operational modal analysis techniques is that the stabilization diagrams tend to produce non-physical modes. This will be discussed in Section 5 of this paper. Applying another kind of stabilisation diagrams can solve this problem. It will be shown that this new approach will eliminate the non-physical modes. The approach will be validated in two ways. First, its efficiency will be shown by using data generated by an acoustic finite element model. In a second step, the approach will be validated using acoustical measurements.

\section{The concept of transmissibility functions}

Before explaining how transmissibility based OMA works, it should be mentioned that there exist several definitions for transmissibility functions. Ewins defined the transmissibility function $T_{i j}(\omega)$ as follows [8]:

$$
T_{i j}(\omega)=\frac{X_{i} e^{i \omega t}}{X_{j} e^{i \omega t}}
$$

So it is the ratio between the responses at two degrees of freedom (DOFs) $i$ and $j$. Note that it is also a function of the frequency $\omega$. When a system is excited at only one location (e.g. DOF k) the transmissibility function $T_{i j, k}(\omega)$ could be modelled as:

$$
T_{i j, k}(\omega)=\frac{H_{i k}(\omega)}{H_{j k}(\omega)}
$$

This shows already that transmissibility functions do depend on the applied forces.

The concept of transmissibility functions has been generalised by several authors so it can also be used in a multiple degree of freedom systems framework [9-11]. However, for simplicity, in this paper no transmissibility matrices will be used. On the other hand, the model of Ewins will be generalised: a transmissibility function $T_{i j}^{A}(s)$ will be defined for a certain loading case A. This loading case can consist of a single force applied to the structure but it also can consist of multiple forces that are simultaneously exciting the structure.

So, a single reference transmissibility function can be modelled as

$$
T_{i j}^{A}(s)=\frac{X_{i}(s)}{X_{j}(s)}=\frac{\sum_{k=1}^{n} H_{i k}(s) F_{k}(s)}{\sum_{k=1}^{n} H_{j k}(s) F_{k}(s)}
$$

$X_{i}(s)$ is the spectrum of the response in point $i, X_{j}(s)$ is the spectrum of the response in the reference point $j, F_{k}(s)$ is the spectrum of the input signal in point $k$ and $H_{i k}(s)$ is the frequency response function (FRF) between point $k$ and point $i$. Note that for a given loading case A, this single reference transmissibility functions will be unique.

\section{Transmissibility driven OMA}

A thorough study about transmissibility driven OMA has recently been performed [6,7]. For simplicity, this contribution will only focus on single reference transmissibility functions.

The key idea behind the technique is that a set of transmissibility functions becomes independent of the applied forces exactly in the system poles and that their value in the system poles is related to the mode shape values. The 
limit value of the transmissibility function for the Laplace variable $s$ going to the system's poles, $\lambda_{m}$, converges to

$$
\lim _{s \rightarrow \lambda_{m}} T_{i j}^{A}(s)=\frac{\phi_{i m}}{\phi_{j m}}
$$

This is true for all possible loading cases $A, B, \ldots$ Hence, it follows that a transmissibility function (which in general is dependent of the sources) will differ for each loading case, except in the poles. In the poles the transmissibility functions will cross. This is shown in Fig. 1. From this Fig. it can be seen that for this system there will be a pole around $22 \mathrm{~Hz}$.

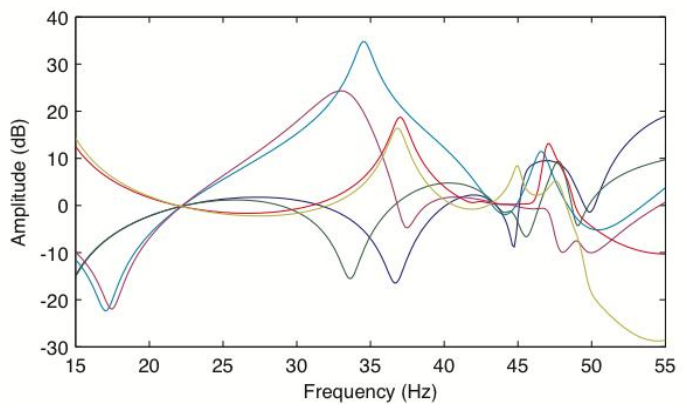

Fig. 1. Single reference transmissibility functions of 6 loading cases.

This also implies that the system's poles, $\lambda_{m}$, are zeroes of the rational function $\Delta T_{i j}^{A B}$, which is defined by

$$
\Delta T_{i j}^{A B}(s)=T_{i j}^{A}(s)-T_{i j}^{B}(s)
$$

It follows that it will be poles of the inverse of $\Delta T_{i j}^{A B}$, i.e.

$$
\Delta^{-1} T_{i j}^{A B}(s)=\frac{1}{\Delta T_{i j}^{A B}(s)}=\frac{1}{T_{i j}^{A}(s)-T_{i j}^{B}(s)}
$$

Using this property of transmissibility functions, it is possible to create rational functions that can be estimated with the classical FRF-estimators to obtain the system poles. Note however that those rational functions contain more poles than the system's poles only [12]. This will cause the classical stabilisation diagrams to show non-physical poles. This will be discussed in section 5 after describing the acoustic system that will be studied in this paper.

\section{Acoustic simulation}

Until now the transmissibility driven OMA procedures have only been tested on mechanical structures. In this contribution it will be applied to an acoustic simulation and to a real acoustic system. The goal is to estimate the modal parameters of the low frequency modes of a small library. Some pictures of the library are shown in Fig. 2.

Acoustic modal analysis is mainly used to model the low frequency sound propagation in cavities. Those cavities can be found, for example, in the transportation industry: in cars, air planes, helicopters, busses and machinery cabins. Other engineering domains where the acoustic modal model is used, are room acoustics, sound field reproduction, noise cancellation and loudspeaker design.

The basic equation for acoustic systems is [13-16]

$$
\left(s^{2} M+s C+K\right) P(s)=s Q(s)
$$



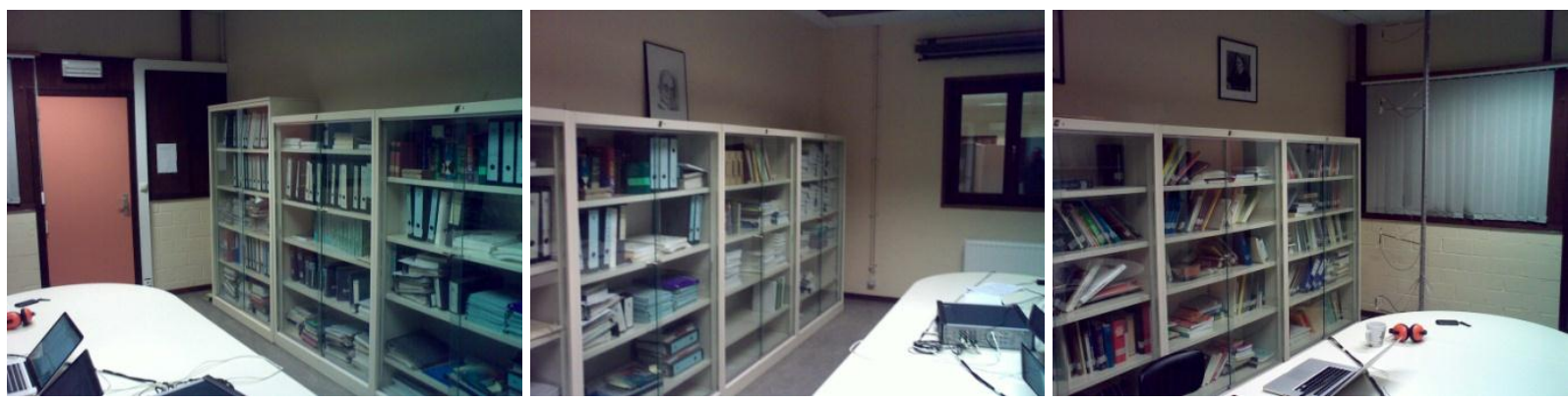

Fig. 2. Acoustic system: Library.

Here $s$ is the Laplace variable, $M$ is the acoustic equivalence of a mass matrix, $C$ is the acoustic equivalence of a damping matrix and $K$ is the acoustic equivalence of a stiffness matrix. $P(s)$ is the pressure vector, i.e. it contains the pressures measured at different DOFs. $s Q(s)$ is the volumetric acceleration vector.

It can be shown that this acoustic model also can be modelled as a modal model [13-16]. However, experimental acoustic modal analysis has one big disadvantage, i.e. the volumetric acceleration of the sound source should be measured [17]. The operational acoustic modal analysis technique does not suffer from this problem [18].

Now, the transmissibility driven OMA procedures will be tested on a Finite Element Model (FEM) which has been created using COMSOL ${ }^{\mathrm{TM}}$. The grid is shown in Fig. 3. The eigenfrequency analysis resulted in 6 modes between $20 \mathrm{~Hz}$ and $50 \mathrm{~Hz}$. They are shown in Fig. 4. The transmissibility driven OMA procedure will be validated using the results of this analysis.

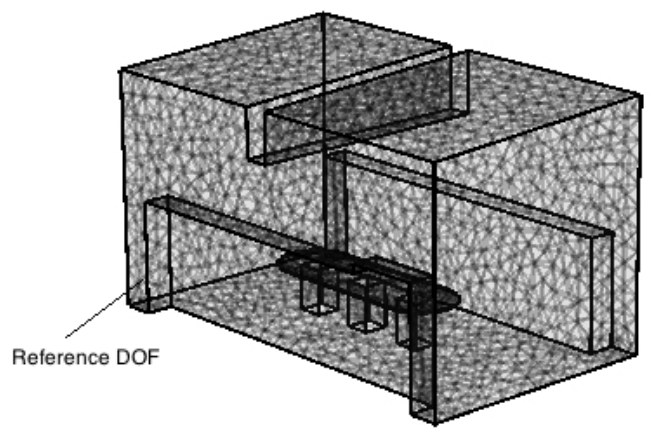

Fig. 3. COMSOL: FEM grid and reference microphone location.

A data set that will be used for validating the transmissibility approach has been created as follows:

- First the exact FRFs were calculated using the COMSOL time-harmonic analysis module.

- Then 6 loading cases have been created by moving a point source to 6 different locations.

- For each loading case, the frequency domain response has been calculated in 144 points (a structured grid with 4 points distributed over the width, 4 points distributed over the height and 9 points distributed over the length of the room). The source signal was normal distributed white noise. For each loading case 4 blocks have been simulated.

- Then the transmissibility matrix and the cross-power matrices were calculated.

\section{Results of the simulations}

Classical Experimental Modal Analysis (EMA), time domain subspace methods and auto/cross-power spectrum driven OMA techniques use a stabilisation diagram to filter the physical poles from the total set of estimated 
poles [19]. In a standard stabilisation diagram the y-axis shows the increasing polynomial order of the estimates. The idea is that the physical poles will show up in each estimate when the chosen order is sufficiently high while the mathematical poles will be different for each chosen order. So, the physical poles will stabilise while the mathematical poles will not stabilise. A typical stabilisation diagram of an EMA is shown in Fig. 5. Here it is obvious that there are 6 modes. Note that this plot results from an EMA using the exact FRFs from the COMSOL simulation.

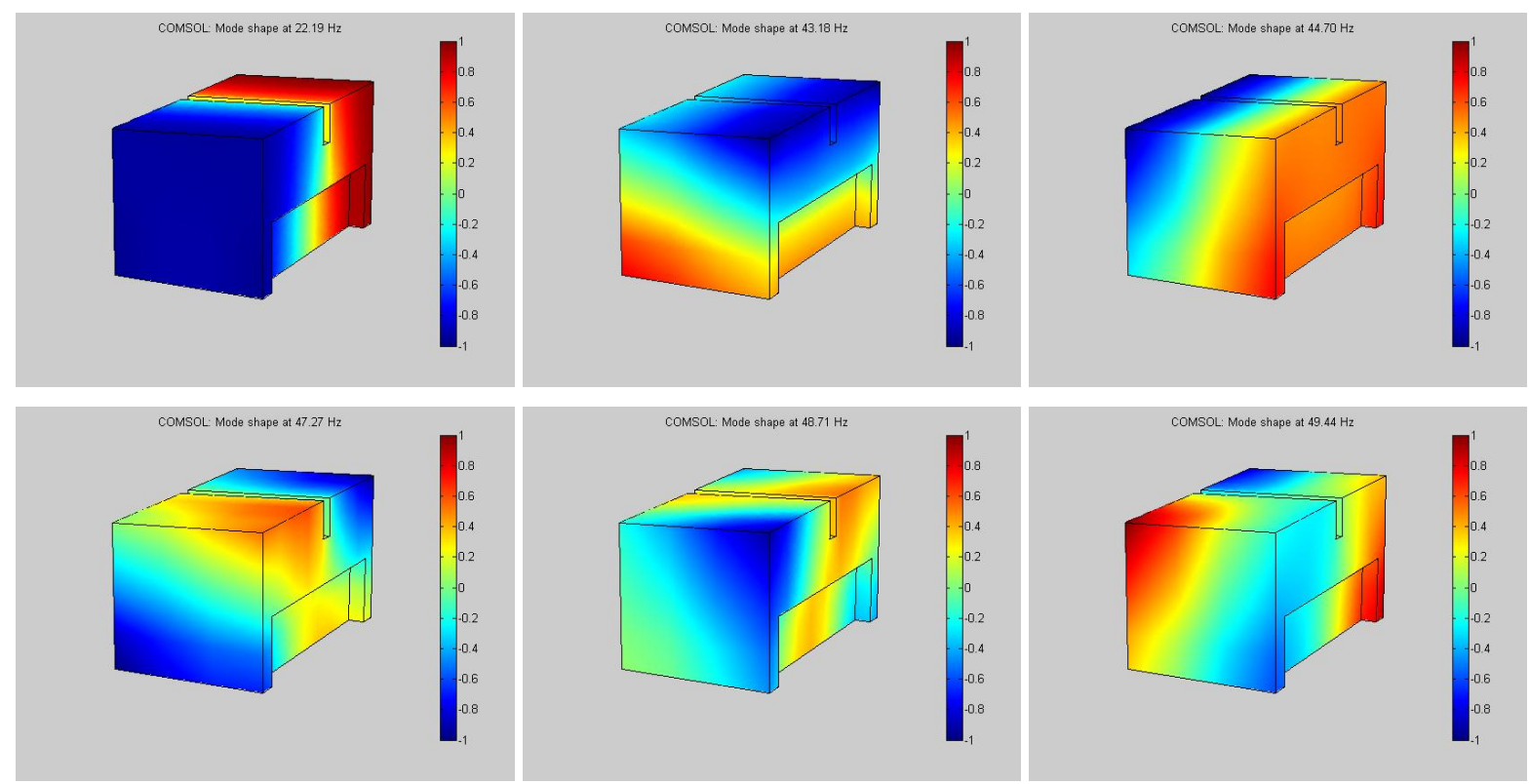

Fig. 4. COMSOL: 6 modes between $20 \mathrm{~Hz}$ and $50 \mathrm{~Hz}$.

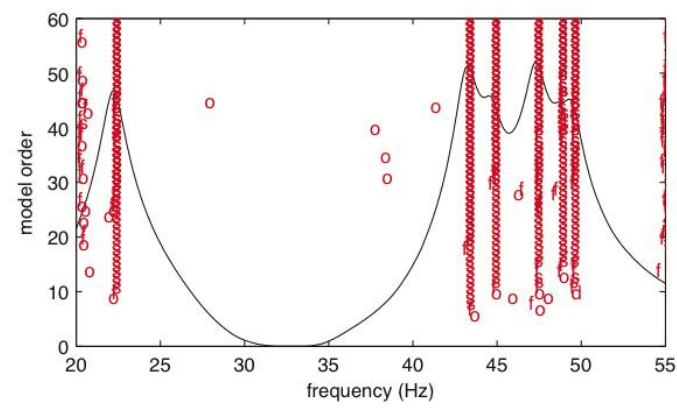

Fig. 5. Stabilisation diagram of an the FRFs (maximum order $=64$ ).

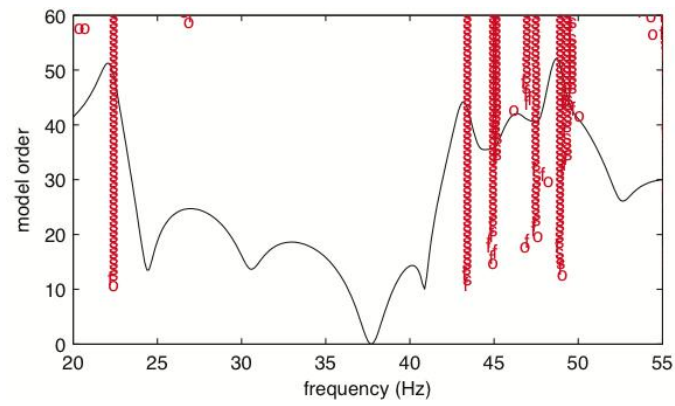

Fig. 6. Stabilisation diagram of a Transmissibility driven OMA (maximum order $=64)$. 
A problem now arises when applying those stabilisation diagrams to transmissibility driven OMA estimates. Figure 6 shows the standard stabilisation diagram applied to the transmissibility driven OMA where all 6 loading cases were combined. From the figure it can be seen that some non-physical poles will probably be selected.

The existence of the non-physical poles can be explained. In a previous section it has been mentioned that transmissibility driven OMA is based on the fact that all single reference transmissibility functions between 2 points evaluated in the poles do intersect. This applies for all loading cases. Figure 7a shows for example some transmissibility functions and it is clear that there will be a physical pole around $22 \mathrm{~Hz}$. On the other hand: when several transmissibility functions intersect the algorithm will try to fit a physical pole at that frequency in the stabilisation diagram. For example: looking at Fig. $7 \mathrm{~b}$ (which plots the transmissibility functions of loading cases 1, 5 and 6), one might expect that at $36 \mathrm{~Hz}$ there will be a stable pole. But if also loading cases 2, 3 and 4 are plotted; it is obvious that there will be no stable pole at $36 \mathrm{~Hz}$. This is shown in Fig. 7a.

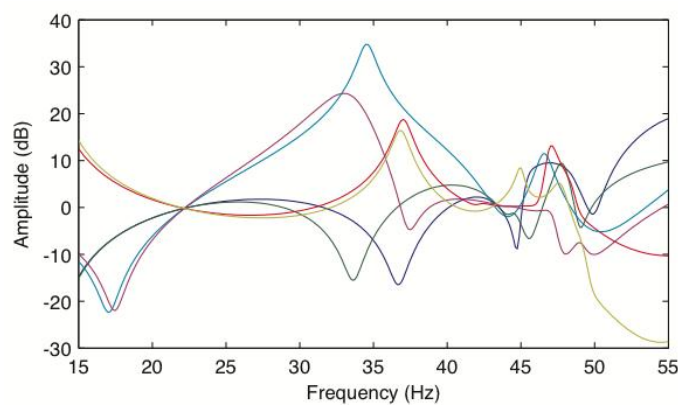

(a) loading cases 1 to 6

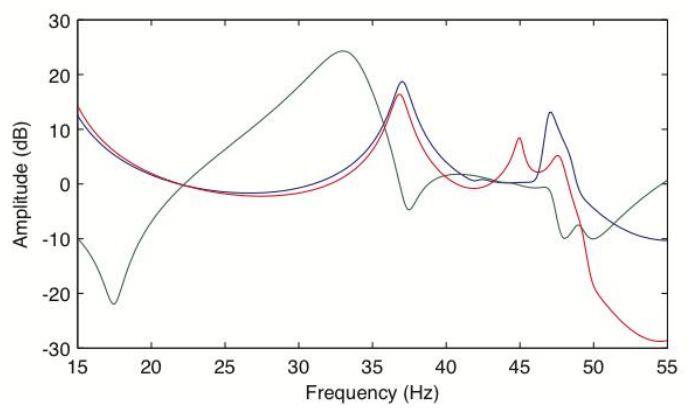

(b) loading cases 1,5 and 6

Fig. 7. Transmissibility functions between 2 points.

The appearance of those fake poles is due to a specific combination of loading cases and they will not disappear with increased order of the estimate. Note that different combinations of loading cases, results in different fake poles. This is shown in Fig. 8. But as has been shown in Fig. 6, even when combining all loading cases, there will still be fake poles.

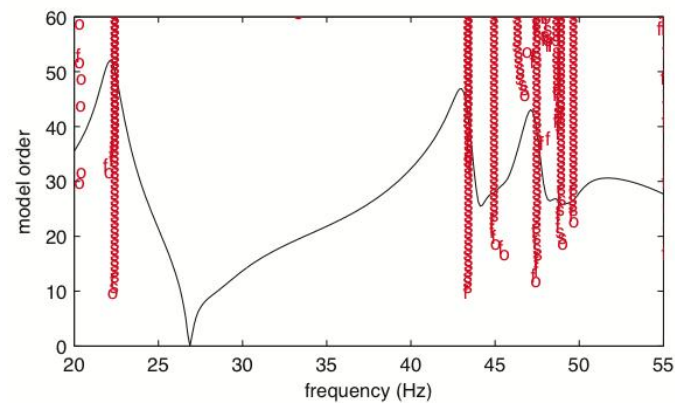

(a) loading cases 1 and 2

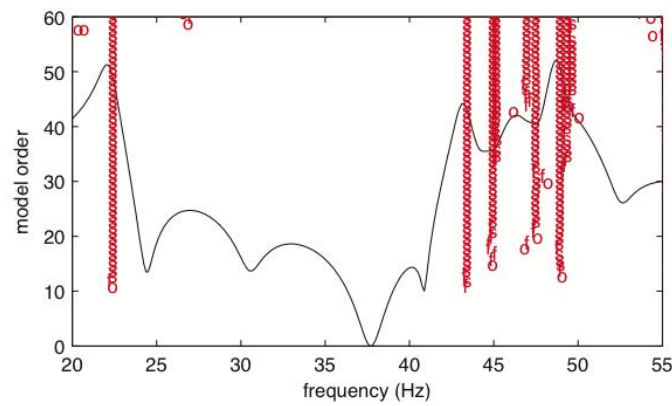

(b) loading cases 5 and 6

Fig. 8. Transmissibility driven stabilisation diagrams: Combining different loading cases.

Note also that even without solving the problem of the fake poles, the stabilisation diagram of the transmissibility driven OMA already is much better than the stabilisation diagram of the auto/cross-power driven OMA. This is shown in Fig. 9 where the stabilisation diagram resulting from the exact FRFs is compared to the transmissibility technique and the Auto/cross-power technique. The stochastic character of the cross-power functions, results in wrongly estimated poles. Especially the damping factors are wrong (they become very small). 


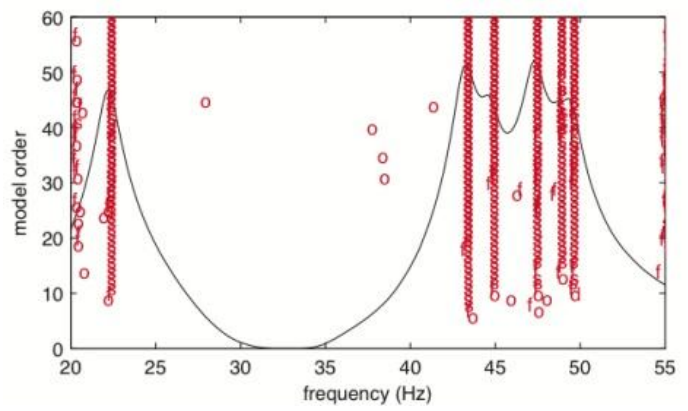

(a) FRFs

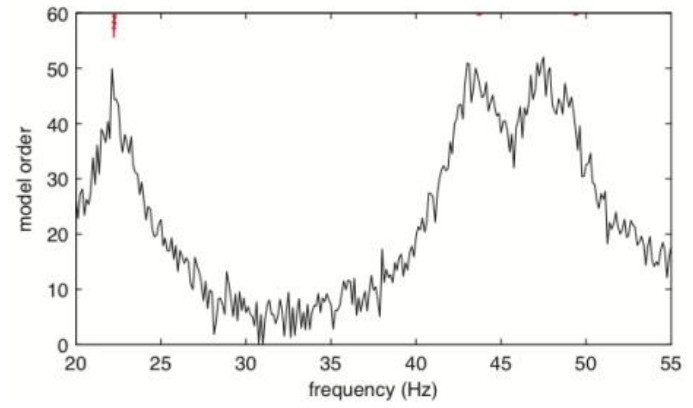

(b) Cross-power driven OMA

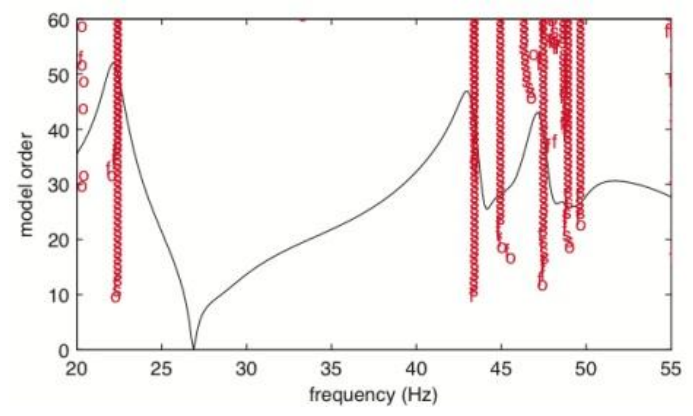

(c) Transmissibility driven OMA

Fig. 9. Stabilisation diagram for loading cases 1 and 2.

Since the fake poles are different for each combination of loading cases, another approach for finding the physical poles is possible. The proposed approach is as follows:

- First a set of combinations of loading cases is created, e.g. all combinations from 3 out of 6 loading cases, 4 out of 6 loading cases and 5 out of 6 loading cases.

- In a second step, on every combination, an OMA estimate is performed.

- Finally, a 2-dimensional histogram is created using all the estimated stable poles.

Since the physical poles should be found in each estimate, they will form big clusters. The fake poles will only form very small clusters. Those clusters can be visualised by creating a 2-dimensional histogram. The first dimension is the damped resonance frequency and the second dimension is the damping ratio. The third dimension shows the amount of stable poles that were found in each interval. The enhanced stabilisation diagrams of our acoustic simulation are shown in Figs 10-12. In Fig. 10, the results for the FRFs are shown, in Fig. 11 the results of the

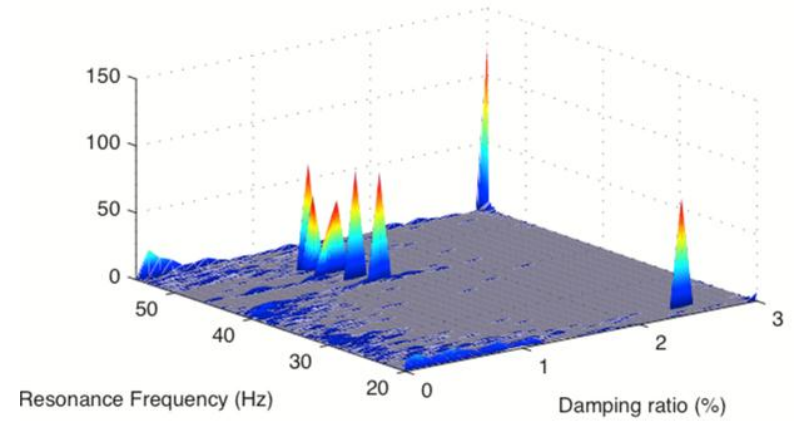

Fig. 10. Clusters of estimated poles from FRFs. 
transmissibility approach are shown and in Fig. 12 the results of the auto/cross-power approach are shown. For the transmissibility driven OMA, it is now much easier to select the physical poles. Note that there are still smaller clusters that are due to the fake poles, but they are significant smaller than the clusters of the physical poles.

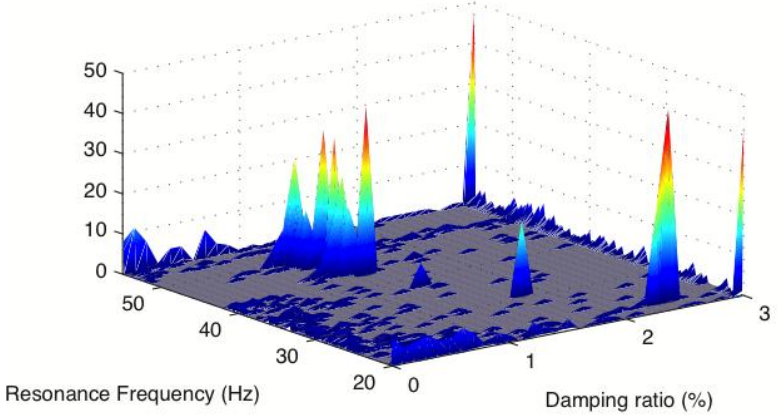

Fig. 11. Clusters of estimated poles from Tranmissibilities.

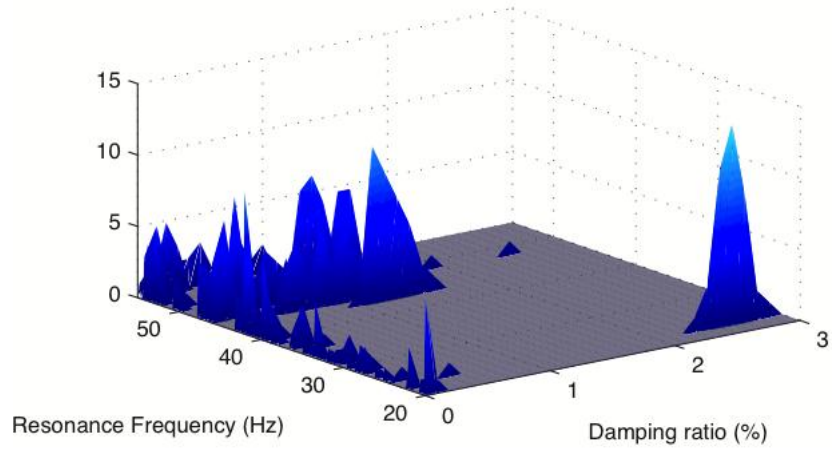

Fig. 12. Clusters of estimated poles from Auto/cross-powers.

Figures 13 and 14 show the results from the cluster analysis. Also these results show that the transmissibility approach results in better estimates compared to the auto/cross-power approach.
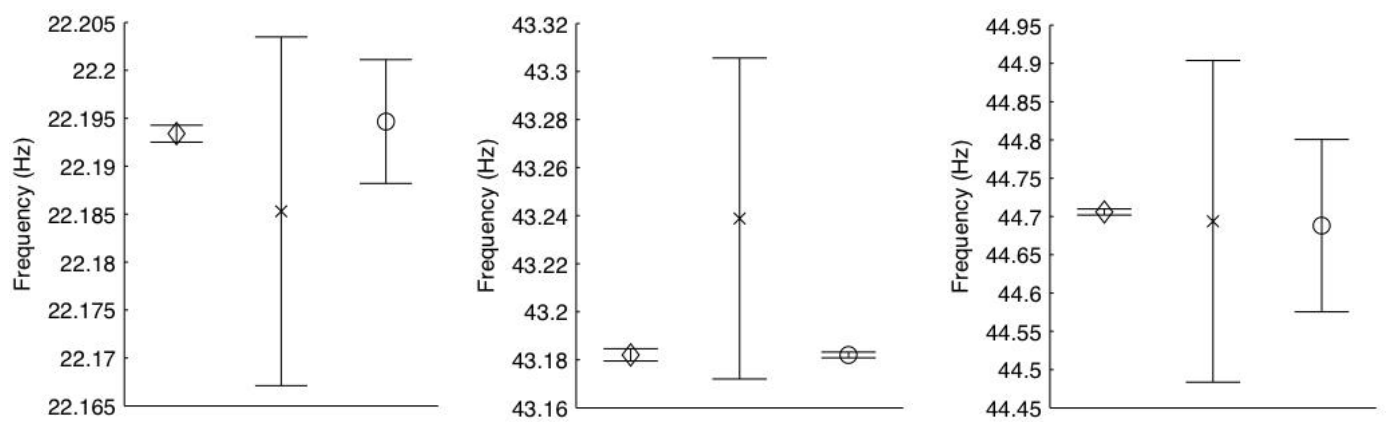

Fig. 13. Results of cluster analysis: Damped resonance frequencies.

\section{Experimental results}

In the previous section it has been shown that the transmissibility approach results in better estimates compared to the cross-power based approach. Also it has been shown how to eliminate the fake poles that always show up using 
the transmissibility approach. In this section, the approach will be validated using real acoustical data. Some pictures of the room can be found on Fig. 2.

A data set that will be used for validating the transmissibility approach has been created as follows:

- First the exact FRFs were measured using 4 roving microphones, and the electrical signal of the loudspeaker as input. The microphones and loudspeaker are shown on Fig. 15.

- 6 loading cases have been created by moving a loudspeaker to 6 different locations.

- For each loading case, the frequency domain response has been calculated in 144 points (a structured grid with 4 points distributed over the width, 4 points distributed over the height and 9 points distributed over the length of the room). The source signal was normal distributed white noise. For each loading case 3 blocks have been measured.

- Then the transmissibility matrix and the cross power matrices were calculated.
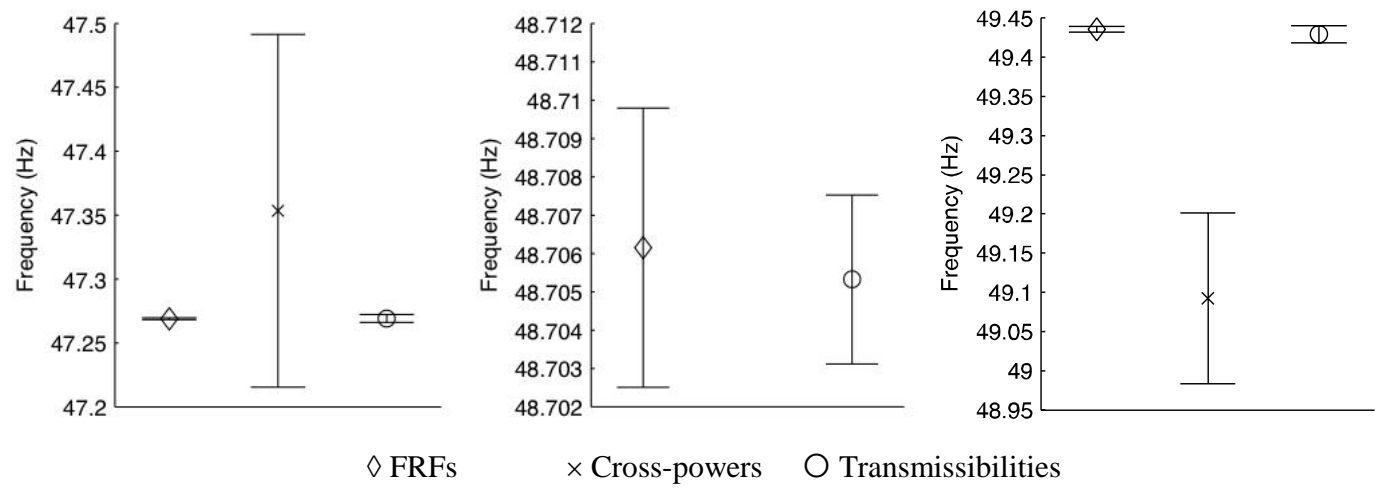

Fig. 14. Results of cluster analysis: damped resonance frequencies.
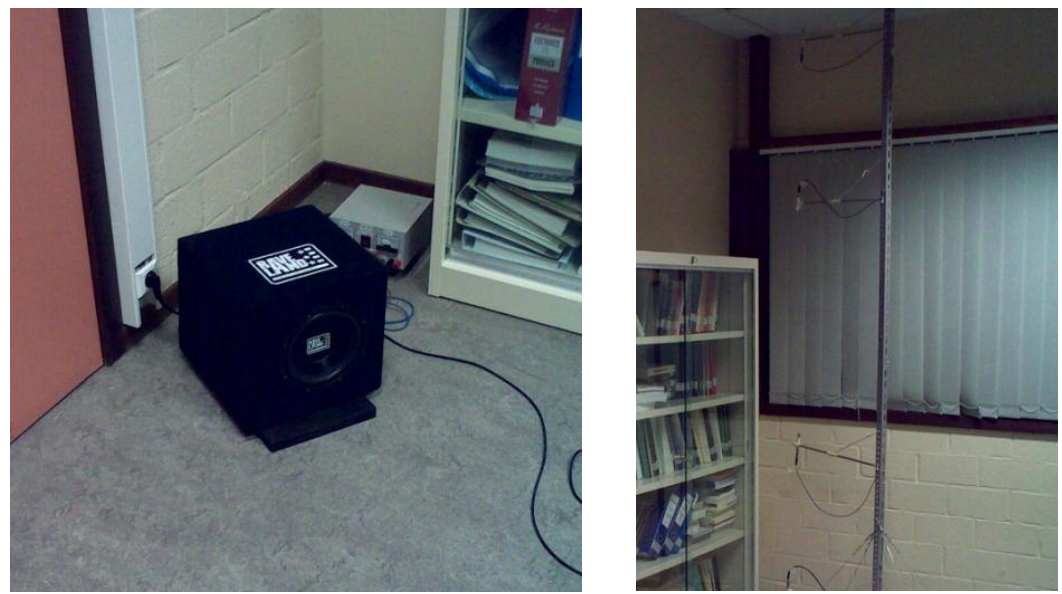

Fig. 15. Loudspeaker and microphones.

The 2-dimensional stabilisation diagrams are shown in Figs 16-18. The mode shapes that resulted from the FRFs are shown in Fig. 19.

The first thing to note is that the cross-power based diagram (Fig. 18) is of much lower quality. The FRF-based diagram gives the best results. Although there are 6 modes, it only shows 5 peaks. However by zooming in and by adapting the number of intervals for the histogram, one would clearly see the 6 different peaks. Note that the peak around $40 \mathrm{~Hz}$ in the shown diagram is higher compared to the other peaks. The reason is that there are two modes which do not differ a lot in damping and frequency. Note also that the same peaks can be found in the transmissi- 
bility approach. However, the estimates are less accurate. This could have been expected since for the FRFs the electrical signal of the loudspeaker has been used as input while the reference used for the transmissibility approach was a fixed reference microphone. Therefore the transmissibility approach cannot separate the poles of modes 3 and 4. This is also shown on Fig. 20. A second reason why the transmissibility approach did not identify mode 4 that well, is that the fixed reference microphone was located in a nodal line of the mode, i.e. the left corner on the floor.

If one compares the results of the measurements with the results of the simulation one notes some differences. The mode shapes of the higher modes do differ. This is probably due to the fact that the geometry which was used for the simulations, was not sufficiently accurate. Also it can be noted that the simulation only took into account the acoustical effects. There was no interaction with the structures in the room. However the first two modes of the measured mode shapes have a high correlation. Probably the first mode is a coupled vibration mode. The lower damping also supports this assumption.

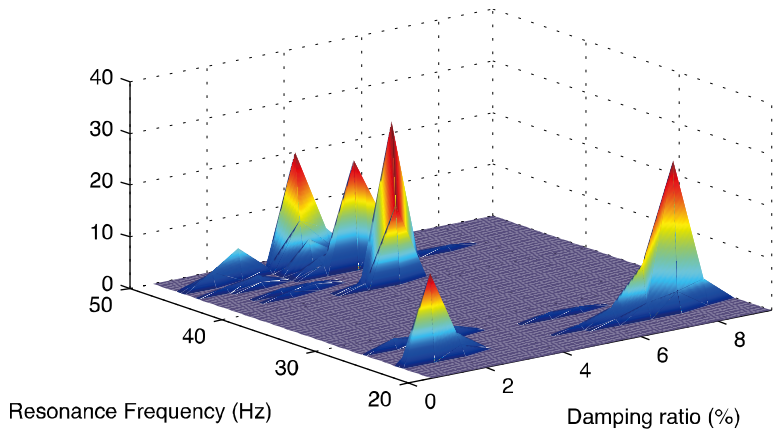

Fig. 16. Clusters of estimated poles from FRFs.

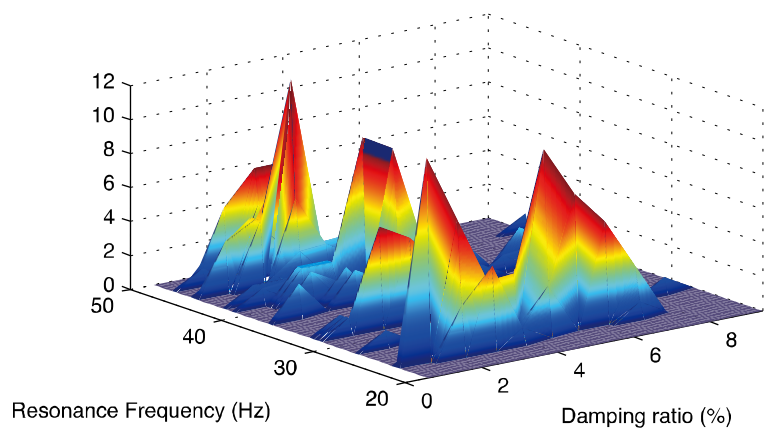

Fig. 17. Clusters of estimated poles from Transmissibilities.

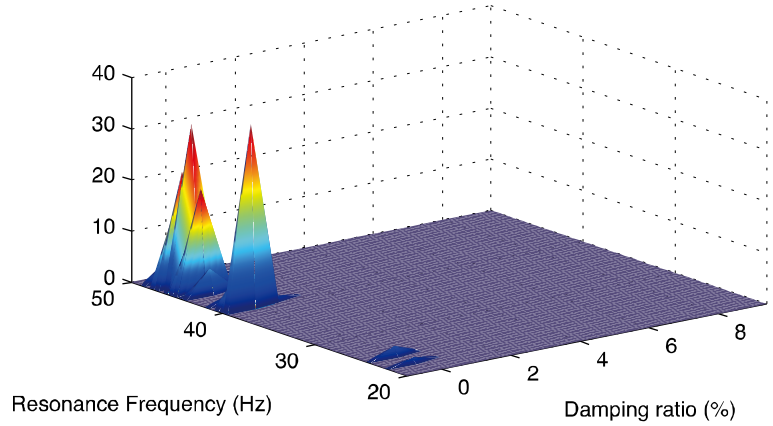

Fig. 18. Clusters of estimated poles from Auto/cross-powers.

\section{Conclusions}

In this contribution the transmissibility driven OMA has been applied for the first time on an acoustic system, i.e. a small library. It has been shown that the results are better than the results obtained with an auto/cross-power driven OMA.

Another problem that has been discussed is the existence of fake poles in the standard stabilisation diagrams when using those diagrams with estimates resulting from a transmissibility driven OMA. A solution to this problem has been proposed. An enhanced stabilisation diagram has been created by generating a 2-dimensional histogram using a set of estimated poles where each estimate is the result of a transmissibility driven OMA which on itself results from a unique combination of loading cases. Since each estimate uses a unique combination of loading cases, the fake poles will be different for each estimate while the physical poles resulting from two estimates will not differ significantly. 


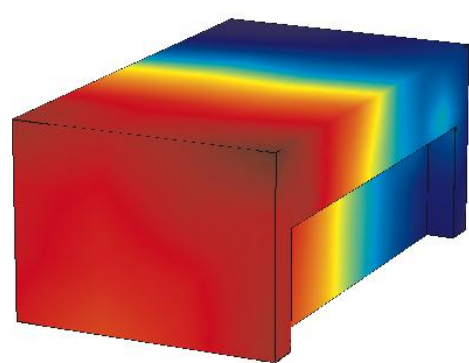

mode 1

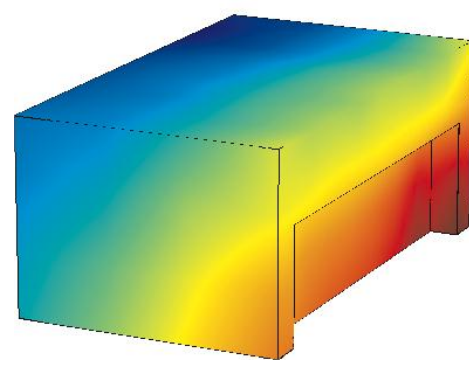

mode 4

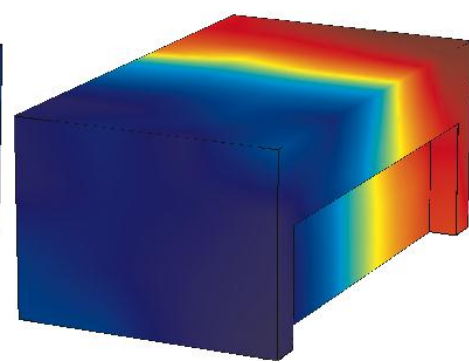

mode 2

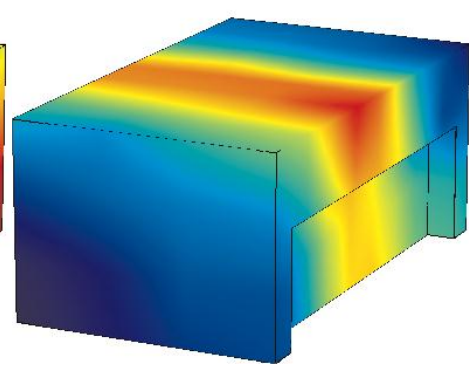

mode 5

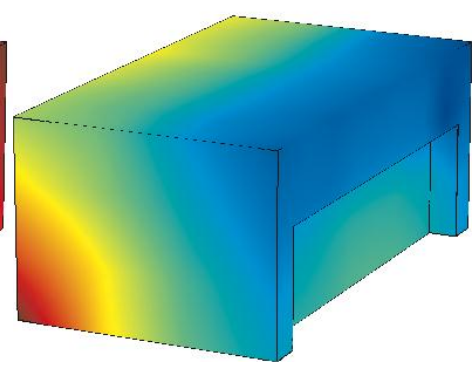

mode 3

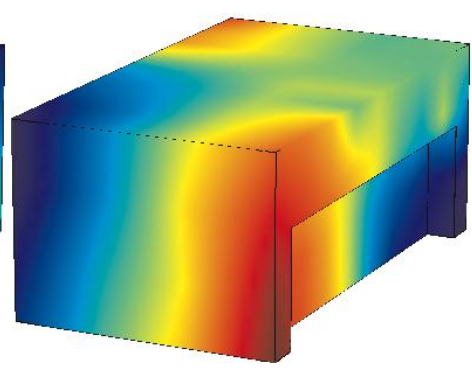

mode 6

Fig. 19. Measured mode shapes of the library.

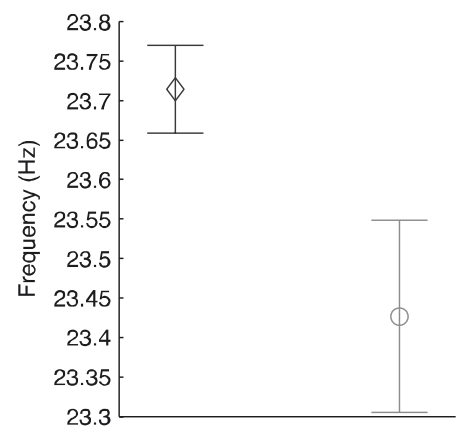

mode 1

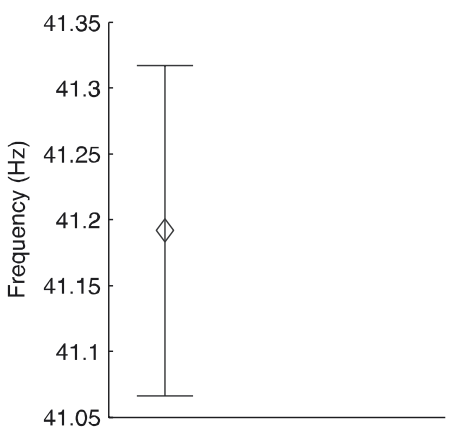

mode 4

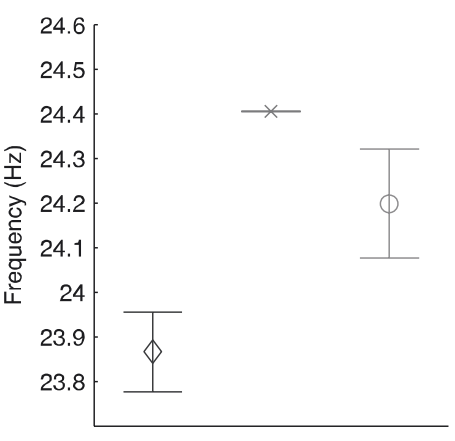

mode 2

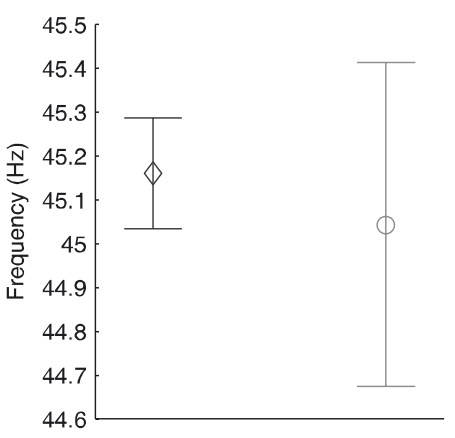

mode 5

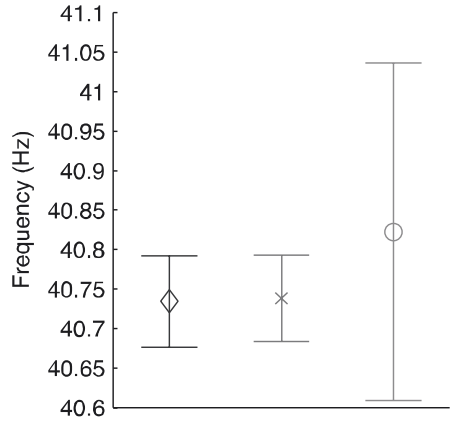

mode 3

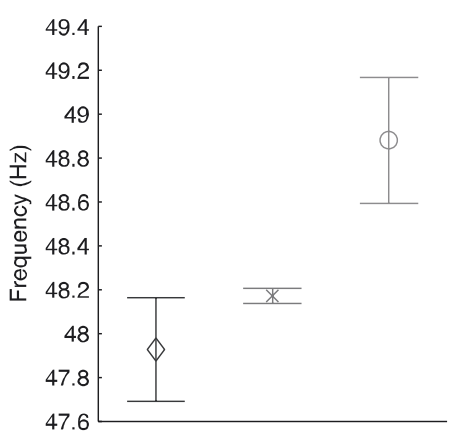

mode 6

$\diamond$ FRFs $\times$ Cross-powers $\bigcirc$ Transmissibilities

Fig. 20. Results of cluster analysis: damped resonance frequencies. 


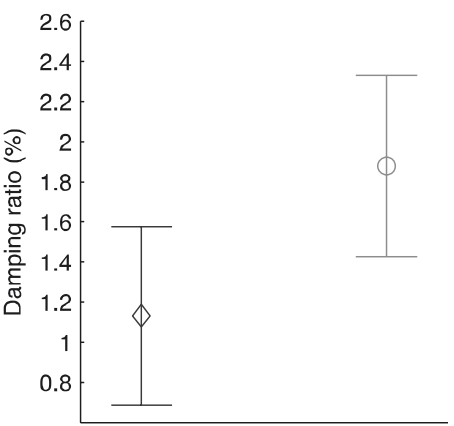

mode 1

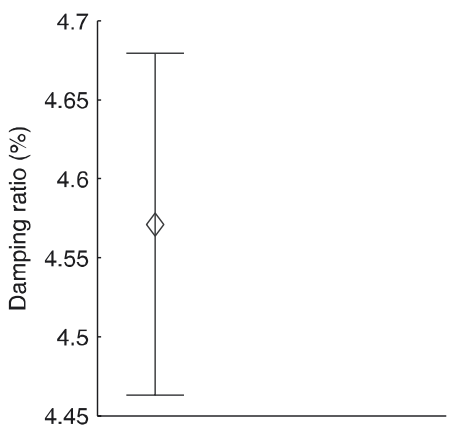

mode 4

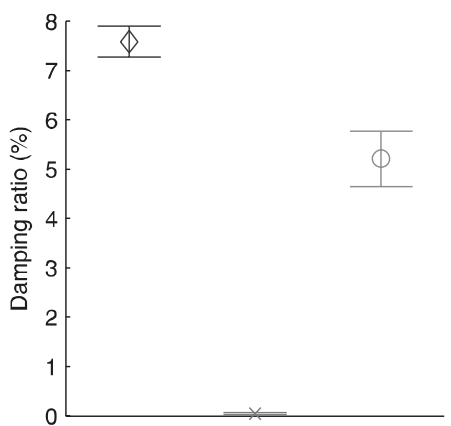

mode 2

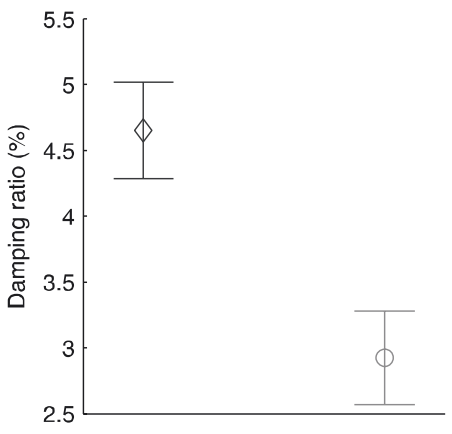

mode 5

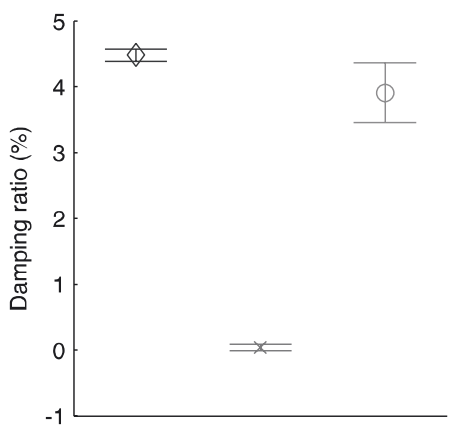

mode 3

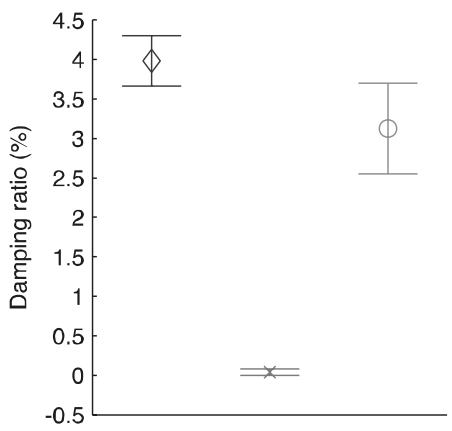

mode 6

$\diamond$ FRFs $\quad \times$ Cross-powers $\bigcirc$ Transmissibilities

Fig. 21. Results of cluster analysis: damping factors.

The proposed method has been applied to the acoustical system, which resulted in accurate estimates of the modes.

\section{References}

[1] L. Hermans and H. Van der Auweraer, Modal testing and analysis of structures under operational conditions: Industrial applications, Mechanical Systems and Signal Processing 13(2) (1999), 193-216.

[2] B. Peeters and G. De Roeck, Stochastic system identification for operational modal analysis: A review, Journal of Dynamic Systems Measurement and Control-transactions of the ASME 123(4) (2001), 659-667.

[3] R. Brincker, C.E. Ventura and P. Andersen, Why Output-Only Modal Testing is a Desirable Tool for a Wide Range of Practical Applications, Proceedings of the 21st International Modal Analysis Conference, Kissimmee (Florida), February 3-6, 2003.

[4] R. Brincker, L. Zhang and P. Andersen, Modal identification of output-only systems using frequency domain decomposition, Smart Materials \& Structures 10(3) (2001), 441-445.

[5] L. Ljung, System identification: Theory for the user, Prentice-Hall information and system sciences series, 1999.

[6] C. Devriendt, On the use of transmissibility functions in operational modal analysis, PhD thesis, Vrije Universiteit Brussel, Dept. of Mechanical Engineering, ISBN 9789054877301.

[7] C. Devriendt, G. De Sitter, S. Vanlanduit and P. Guillaume, Operational modal analysis in the presence of harmonic excitations by the use of transmissibility measurements, Mechanical Systems and Signal Processing 23(3) (2009), 621-635.

[8] D.J. Ewins, Modal Testing: Theory, Practice and Application, Wiley, 2001.

[9] A. Ribeiro, J. Silva and N. Maia, On the generalisation of the transmissibility concept, Mechanical Systems and Signal Processing 14 (1) (2000), 29-35.

[10] N. Maia, J. Silva and A. Ribeiro, The transmissibility concept in multi-degree-of-freedom systems, Mechanical Systems and Signal Processing 15(1) (2001), 129-137.

[11] M. Fontul, A. Ribeiro, J. Silva and N. Maia, Transmissibility matrix in harmonic and random processes, Shock and Vibration 11(5) (2004), 563-571.

[12] C. Devriendt, T. De Troyer, G. De Sitter and P. Guillaume, Automated operational modal analysis using transmissibility functions, Proceedings of ISMA 2008: International Conference on Noise and Vibration Engineering, Leuven (Belgium), 2008, pp. 2457-2469. 
[13] P. Filippi, Theoretical acoustics and numerical techniques, Courses and lectures - International Centre for Mechanical Sciences, Springer-Verlag, 1983.

[14] A. Craggs, Use of simple 3-dimensional acoustic finite elements for determining natural modes and frequencies of complex shaped enclosures, Journal of Sound and Vibration 23(3) (1972), 331-339.

[15] P. Sas and F. Augusztinovicz, Acoustic modal analysis, NATO Advanced Study Institute Series E applied sciences (363) (1999), 487-506.

[16] W. Desmet and D. Vandepitte, Finite element method in acoustics, in Proceedings of ISAAC 12 (Advanced Techniques in Applied and Numerical Acoustics), 2001.

[17] K. Wyckaert and L. Meulewaeter, On the influence of finite acoustic source dimensions on acoustical frequency response functions inside an enclosed cavity, in Proceedings of the International Conference on Noise and Vibration Engineering (ISMA-21), 1996.

[18] G. De Sitter, P. Guillaume, S. Vanlanduit and C. Devriendt, Operational acoustic modal analysis: Sensitivity-based mode shape normalisation, Acta Acustica United With Acustica 94(4) (2008), 580-587.

[19] J. Cooper, Comparison of modal parameter-estimation techniques on aircraft structural data, Mechanical Systems and Signal Processing 4(2) (1990), 157-172. 

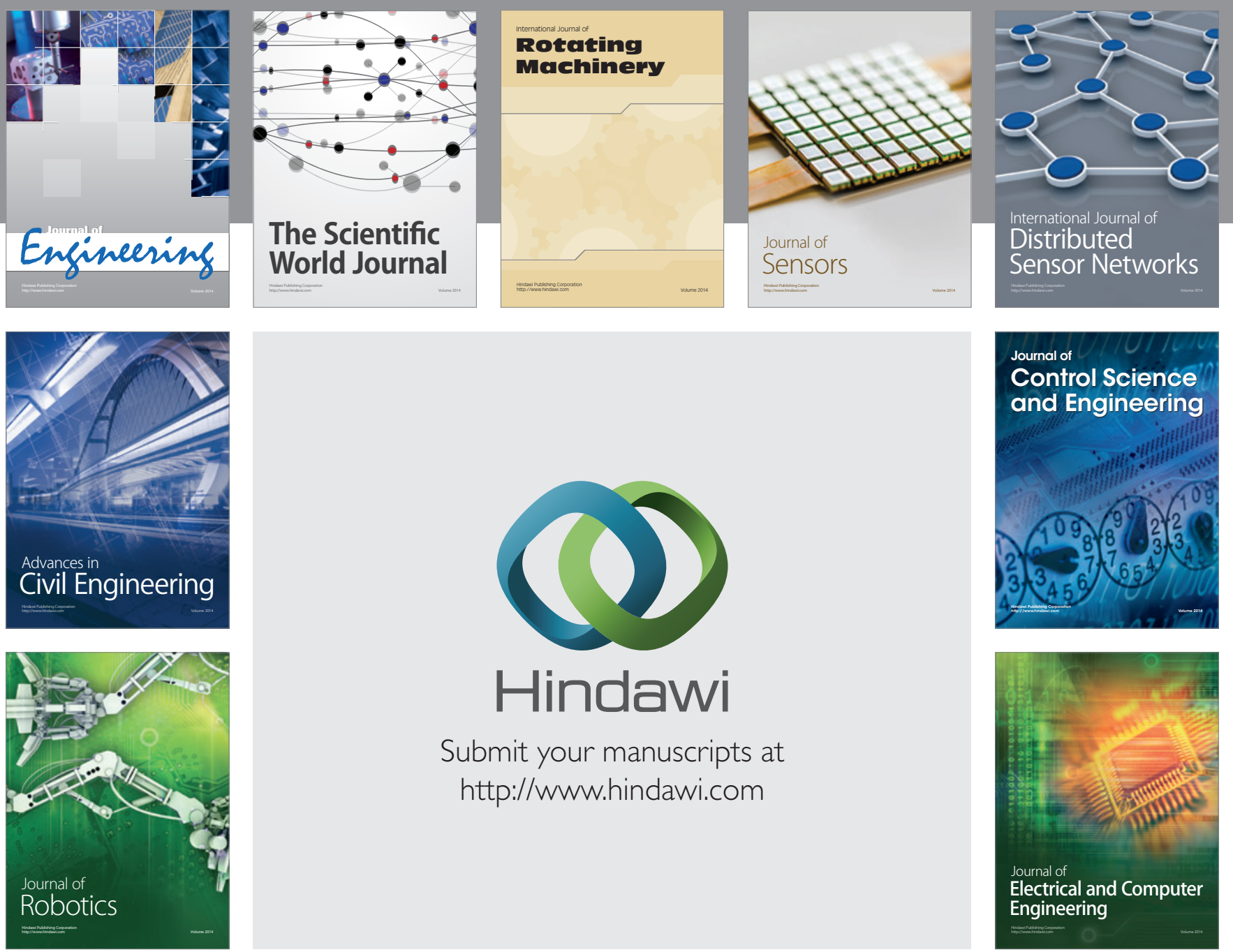

Submit your manuscripts at

http://www.hindawi.com
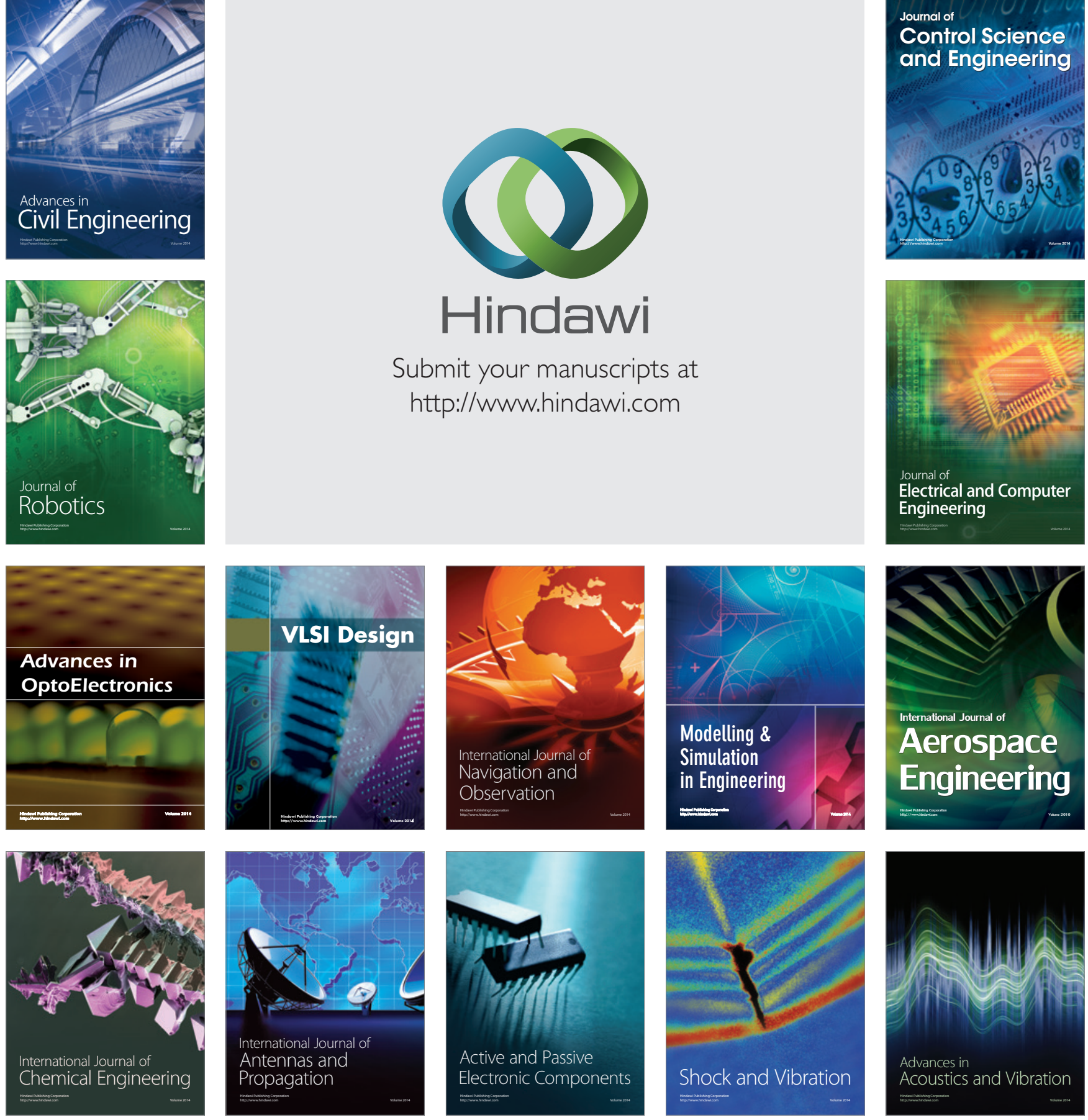